\title{
ESTIMACIÓN POBLACIONAL Y DAÑO AGRÍCOLA DE Lepus europaeus (LIEBRE EUROPEA) EN ÉPOCA LLUVIOSA EN LA PENÍNSULA DE CHUCUITO, LAGO TITICACA, PERU
}

\section{POPULATION ESTIMATE AND AGRICULTURAL DAMAGE OF Lepus europaeus (LIEBRE EUROPEA) IN RAINY SEASON IN THE CHUCUITO PENINSULA, LAKE TITICACA, PERÚ}

\author{
Claudia Beatriz Ramos Nina ${ }^{1}$ y Ángel Canales Gutiérrez ${ }^{2}$
}

\begin{abstract}
Resumen
La investigación realizamos en las comunidades: Churo, Karina y Luquina Chico, ubicada en la península de Chucuito - Puno, Perú, entre diciembre 2010 y mayo 2011. Los objetivos fueron: 1) Evaluación del tamaño poblacional de Lepus europaeus (liebre europea), 2) Verificación de daños ocasionados en la producción agrícola en tres comunidades, 3) Determinación del método de captura adecuada para disminuir y controlar las poblaciones de liebre europea. Aplicamos el método de transectos lineales, utilizada para determinar el tamaño poblacional y el método de observación indirecta de huellas, a través de estaciones olfativas, con registro de huellas. Colocamos 160 estacas para trampas Lazo en total para las comunidades en estudio, alrededor de las cuales colocamos 100 estaciones olfativas; asimismo realizamos Chacu de liebres (captura con participación de varias personas). Finalmente, cuantificamos el porcentaje de pérdidas económicas a través de observaciones in situ en 10 parcelas/comunidad. Con base en el área consumida por liebre del total del área/parcela, aplicamos porcentaje de daño y realizamos entrevistas. En base al método de trampas Lazo y Chacu, comparamos el método adecuado de captura. Los resultados fueron: poblacional estimada de $1.2 \mathrm{ind} / \mathrm{ha}$ en Churo, $1.1 \mathrm{ind} / \mathrm{ha}$ en Karina y $2.4 \mathrm{ind} / \mathrm{ha}$ en Luquina Chico, mientras que la abundancia obtenida en las estaciones olfativas en 5 cultivos diferentes fue: 11.7 individuos en Luquina Chico, 10.8 individuos en Churo y 9.3 individuos en Karina. En el análisis de pérdidas económicas, se obtuvo que: a mayor daño económico existe mayor costo $(\mathrm{r}=0.67)$ entre 2 variables evaluadas en Churo ( $r=0.64)$ en Karina y $(r=0.49)$ en Luquina Chico. Finalmente demostramos que no hay diferencia entre los métodos de captura trampas Lazo y Chacu $\left(\mathrm{H}_{\text {calc }(0.05)}=1.19 ; 1 \mathrm{gl} ; \mathrm{p}=0.2000\right)$.
\end{abstract}

Palabras clave: agrícola, captura, daños, estaciones, huellas, liebre europea, población, transectos.

\begin{abstract}
The research was carried out in the communities: Churo, Karina and Luquina Chico, located in the Chucuito - Puno peninsula, Peru, from december 2010 to may 2011. The objectives were: 1) Evaluation of the population size of Lepus europaeus (European hare), 2) Verification of damages caused in agricultural production in three communities, 3) Determination of the appropriate method of capture to reduce and control the populations of European hares. We apply the linear transect method, used to determine the population size and the method of indirect observation of fingerprints, through olfactory stations, with fingerprints. We placed 160 stakes for loop traps in total for the communities under study, around which we placed 100 olfactory stations, and we also made Chacu de hares (capture with the participation of several people). Finally, we quantified the percentage of economic losses through on-site observations in 10 plots / community. Based on the area consumed by hare of the total area / plot, we apply damage percentage and conduct interviews. Based on the loop and Chacu trap method, we compared the appropriate method of capture. The results were: estimate population of $1.2 \mathrm{ind} / \mathrm{ha}$ in Churo, $1.1 \mathrm{ind} / \mathrm{ha}$ in Karina and $2.4 \mathrm{ind} / \mathrm{ha}$ in Luquina Chico, while the abundance obtained in the olfactory seasons in 5 different crops was: 11.7 individuals in Luquina Chico, 10.8 individuals in Churo and 9.3 individuals in Karina. In the analysis of economic losses, it was found that: the greater the economic damage, the higher the cost $(r=0.67)$ between two variables evaluated in Churo $(r=0.64)$ in Karina and $(r=0.49)$ in Luquina Chico. Finally, we show that there is no difference between trap methods Lazo and Chacu $\left(\mathrm{H}_{\text {calc }(0.05)}=1.19 ; 1 \mathrm{gl}\right.$; $\mathrm{p}=0.2000$ ).
\end{abstract}


Key words: farming, seasons, catch, damage, fingerprints, population, European hare, damages, transects, stations and tracks.

\section{Introducción.}

Esta especie fue reportada formalmente en el Perú desde el año 2002 para Tacna y Arequipa (Cossios 2004; Lleellish et al., 2007). Posteriormente su distribución fue ampliada para los alrededores del lago Titicaca en Puno y la puna sur de Cusco, tanto para localidades alto andinas por debajo de $4400 \mathrm{~m}$, como para la costa de Tacna (Canales, 2008; Bonino et al., 2010), asimismo la liebre europea en el Perú, al 2011, presenta una amplia distribución en el altiplano y la costa sur, registrándose en ecosistemas abiertos de los departamentos de Arequipa, Cusco, Moquegua, Puno y Tacna) (Zeballos et al., 2012), sin embargo la presencia de la liebre se registró por primera vez en los departamentos de Moquegua y Puno, este último ubicado al este del lago Titicaca y en el límite con Bolivia, en general la distribución de la liebre europea abarca los territorios de Argentina y Chile, el sudeste de Perú, el sudoeste de Bolivia, el sudeste de Paraguay y el centro-sur de Brasil (Bonino et al., 2010).

Por tal motivo existe una migración permanente de Lepus europaeus (liebre europea) hacia los ecosistemas alto andinos donde sus condiciones geográficas les permiten realizar campañas de siembra entre 01 a 02 veces al año. La liebre europea por sus hábitos herbívoros compite con el ganado y los mamíferos nativos por los pastos. Se ha reportado que en Patagonia Argentina causan perjuicios a diferentes tipos de producciones agropecuarias (Bonino et al., 2010), la liebre europea es uno de los ejemplos más preocupantes para la conservación de los cultivos agrícolas, afecta principalmente los cultivos de ají paprika (Capsicum annuum), en Tacna - Perú (Lleellish et al., 2007).

La liebre europea es una especie introducida e invasora, que amenaza los ecosistemas y hábitat de zonas cultivables, con efectos económicos de consideración los que oscilan entre el $40 \%$ en épocas de lluvia en una campaña de siembra (Zeballos et al., 2012). En la región Puno teniendo mayor frecuencia en la península de Capachica en Perú, esta especie presenta una densidad de 4.3 y 1.5 ind/ha y se han reportado daños en cultivos como la alfalfa en $30 \%$ y la cebada 27\% (Canales, 2008).

La liebre europea se encuentra además de Perú, en Argentina, Bolivia y Brasil, se ha reproducido exponencialmente, cuenta con una alta tasa reproductiva, siendo el período de gestación alrededor de 40 días y cada hembra tiene 2 pariciones por estación y 2 por camada (INTA, 2012), por lo que la disponibilidad de alimento por cultivos agrícolas. La tasa promedio de dispersión de la liebre europea en Perú es aproximadamente de $37 \mathrm{~km} / \mathrm{año}$ (Cossios, 2004), 34 km/año en Brasil (Salomone, 2006), 30 km/año en Bolivia y de 10 a 17 km/año en Paraguay
(Bonino et al., 1997), en Chile, indican que esta especie es parte importante en la dieta del puma (Puma concolor) reduciendo la presión de caza sobre guanacos y pequeños mamíferos nativos, que es una forma natural de controlar la población de liebres (Wiens, 1984).

El objetivo de este estudio fue evaluar la densidad poblacional en de la liebre europea en dos estaciones diferentes del año, verificar los daños en la producción agrícola y determinar el método de captura para disminuir y controlar sus poblaciones, en la Península de Chucuito - Puno, Perú. La originalidad del estudio se fundamenta en registrar y analizar la densidad poblacional en las penínsulas de Capachica (Canales, 2008) y el estudio actual en la península de Chucuito, se analiza comparativamente, las densidades y daños agrícolas.

\section{Materiales y métodos. \\ Zona de estudio}

La investigación fue realizada en tres comunidades cuyas condiciones termodinámicas, por su cercanía a las orillas del lago Titicaca, permitirían tener mayor posibilidad de producción agrícola, dentro de la península de Chucuito - Perú; las cuales son las localidades de: Churo, Karina y Luquina Chico, ubicadas entre las coordenadas: 15'50'16"S, $69^{\circ} 48^{\prime} 20^{\prime}$ O. Las comunidades humanas de la península de Chucuito son eminentemente agropecuarias, tienen extensiones de terreno cultivado de alfalfa (Medicago sativa) que en promedio es de 1 ha/familia. El área de estudio total en la península que comprende las tres comunidades (Churo, Luquina y Karina), es estima en $22.13 \mathrm{~km}^{2}$. Este cálculo se realizó en base a la georreferenciación de las zonas estudiadas (Figura 1).

Estimación de la densidad poblacional (individuos/ha) de liebre europea en la Península de Chucuito - Puno.

Realizamos transectos lineales de $50 \mathrm{~m}$ en época lluviosa, el monitoreo programado se realizó con una frecuencia de una vez al día, el cual tuvo 5 repeticiones semanales; los horarios estipulados realizamos a partir de las 06:00 h hasta las 10:00 h, entendiendo que son especies que se movilizan con mayor frecuencia durante la noche por sus hábitos nocturnos. Mientras que durante el día, la liebre europea descansa entre la vegetación ( $\mathrm{Vu}, 2001)$, el recorrido fue a pie considerando que en esta época de brote en la producción forrajera permite tener mayor presencia de liebre (Cossios, 2004), recorriendo áreas de cultivos y vegetación natural, teniendo en cuenta una longitud y ancho conocido (largo 500 metros, ancho 10 metros en promedio). En cada comunidad la longitud de los transectos varió según la geografía de la zona, 


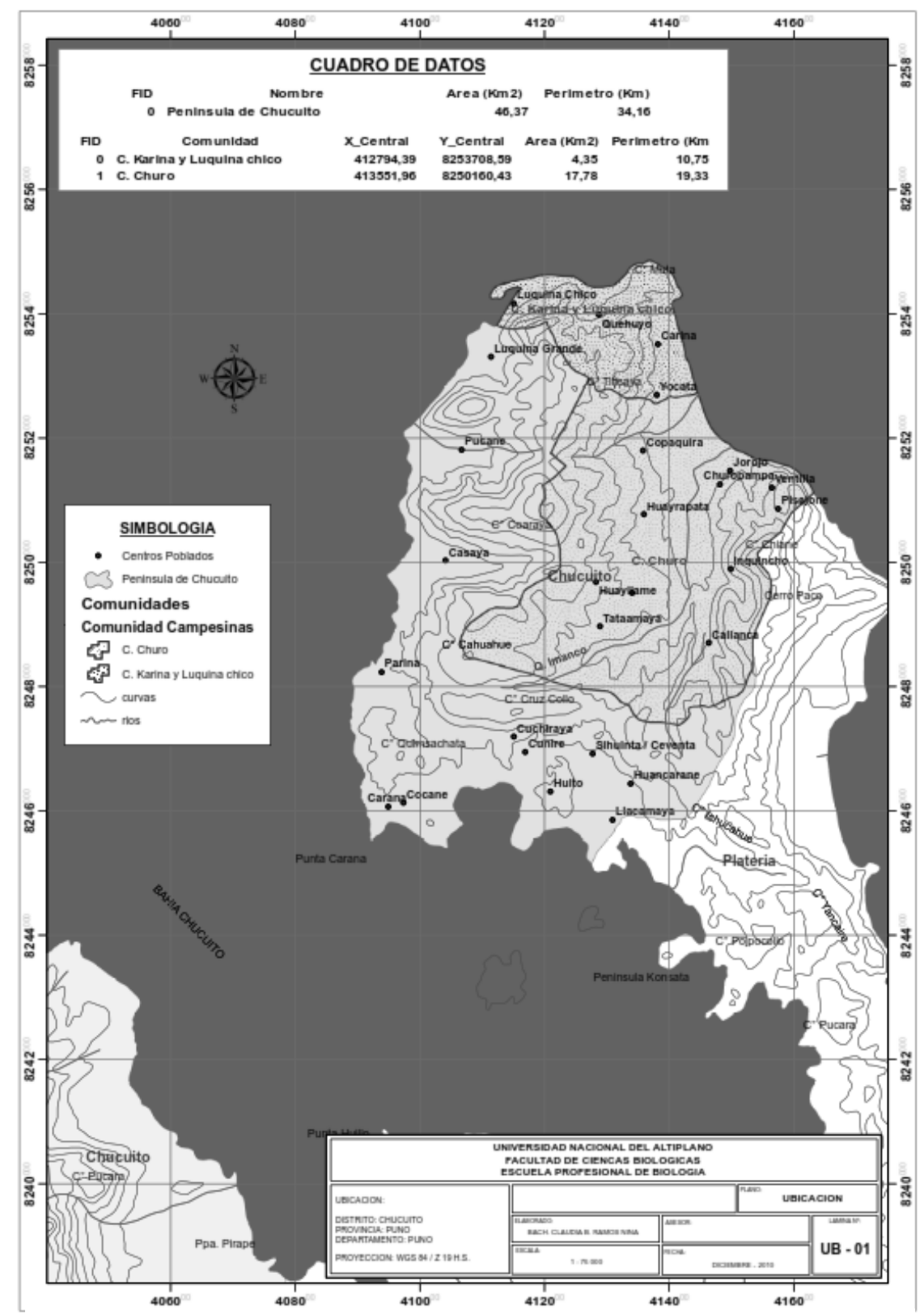

Figura 1. Mapa de ubicación del estudio de liebre europea en las comunidades de Churo, Karina y Luquina Chico de la Península de Chucuito, lago Titicaca, Puno.

reduciéndose el recorrido según los márgenes estipulados en la dato anterior de las medidas de cada transecto. Las observaciones de los individuos de liebre fueron en forma visual directa, realizando una estimación visual de la distancia perpendicular en relación a la línea de transecto, aplicando el estimador Hayne.

Los individuos registramos en una sola dirección del transecto con la finalidad de no duplicar el número de individuos fueron: número de individuos, se estimó en forma visual la distancia perpendicular de la liebre al transecto y longitud del transecto. Aplicamos el estimador de densidad de Hayne (ind./ha), cuya fórmula es: $\mathrm{D}=10^{4} \sum(1 / \mathrm{di}) / 2 \mathrm{~L}$, donde: $\mathrm{D}=$ Densidad, $10^{4}=\mathrm{ha}$; di= distancia de observación del individuo al transecto y $\mathrm{L}=$ Longitud del transecto lineal.

Para el contraste de hipótesis utilizamos la prueba no paramétrica de Kruskal Wallis, que sólo requiere como supuesto la independencia de datos, esta prueba permitió evaluar las diferencias estadísticas entre las densidades poblacionales de la liebre europea entre las distintas comunidades. Para dicho análisis se utilizó el Programa INFOSTAT. 
Estimamos también la abundancia relativa de la liebre europea a través de la instalación de 100 estaciones olfativas para muestreo en cada una de las comunidades de estudio durante seis meses. La frecuencia del monitoreo fue de 1 vez al día con repeticiones de 5 veces por semana en horarios de 05:00 a 09:00 h. La metodología que utilizamos fue adaptar $1 \mathrm{~m}^{2}$ de terreno tamizado de tierra en la periferia de los cultivos. Esta ubicación permite inferir que las liebres ingresaron al cultivo para forrajear y pposteriormente en cada estación olfativa se contabilizó el número de huellas de liebres fijadas en la estación olfativa, observamos la dirección de las mismas, contabilizando solo las que estuvieran orientadas hacia el cultivo y las que salían de los mismos, mas no las que tuvieron direcciones laterales considerándolas inactivas, esto implica que no ingresaron a los cultivos y por ende no consumieron ningún cultivo, entonces, sólo estuvieron de paso, hacia otro cultivo. Las parcelas de cultivos que son afectados por la actividad de consumo de la liebre son: alfalfa, avena, cebada, papa y quinua.

Registramos todas las huellas de la liebre europea a través de moldes colocando mezclas de yeso blanco, teniendo en cuenta si pertenecen a uno, dos o más liebres, teniendo en cuenta la posición de las patas delanteras y traseras con la finalidad de analizar y preservar dichas huellas como evidencia de la presencia de la especie. Incluir las variables consideradas para obtener datos de abundancia y como los manejaron.

Cuantificación del porcentaje de daño agrícola causado en las parcelas de los pobladores de la Península de Chucuito - Puno.

Efectuamos observaciones in situ entre los meses de diciembre 2010 a mayo 2011, teniendo un previo conocimiento sobre el tipo y modo de ramoneo de liebre, en vista de que su presencia es constante en esta comunidad; en ese sentido, se midió el área dañada en cada una de las parcelas que cultivaban las familias, que variaba entre 300 a 1/2 ha, ubicadas en cada una de las tres comunidades evaluadas que poseen cultivos de alfalfa, avena, cebada, papa y quinua. En base al área consumida del tamaño de la parcela por las liebres $\left(\mathrm{m}^{2}\right)$ del total del área de la parcela, previamente identificadas dichas parcelas por la presencia de liebre europea y luego aplicamos porcentajes del daño en los cultivos agrícolas.

Previamente, elaboramos fichas de evaluación para registrar los daños y entrevista a las familias afectadas con preguntas sobre la frecuencia de daños similares que sufrieron por la liebre en sus cultivos. Se realizaron en total 25 entrevistas en las tres comunidades. Las entrevistas fueron focalizadas a cada familia con preguntas sencillas como: presencia de la liebre, daño en los cultivos, que tipos de cultivos, horarios de daños, cantidad de liebre.

Luego, calculamos en forma aleatoria, simulando como ramoneo de liebre en diferentes parcelas qué área $\left(\mathrm{m}^{2}\right)$ correspondía a $1 \mathrm{~kg}$ de peso de alfalfa, avena, cebada, papa y quinua. Teniendo en cuenta ese detalle, medimos el área ramoneada y afectada por la liebre. En base a esa medida calculamos el costo a precio de mercado y la pérdida económica producida en los cultivos. El método estadístico aplicado en esta evaluación fue de correlación de Pearson, donde Y1= costos en soles (variable dependiente); Y2= porcentaje de daño (variable dependiente) y determinamos el grado de asociación entre ambas variables.

Determinación del método de captura de liebre europea adecuado para disminuir y controlar sus poblaciones en la Península de Chucuito - Puno.

Comparamos dos metodologías: trampas Lazo y Chacu. Las trampas Lazo consiste en instalar estacas con nudos corredizos- Instalamos 160 estacas por los alrededores de 10 parcelas con una distancia de 5 metros una estaca de otra. La instalación de las mismas fue desde las 14:00 a 17:00 h. Posteriormente activamos las trampas Lazo haciéndole el nudo corredizo a las 18:00 h, en vista de que las liebres tienen prioritariamente hábitos nocturnos e inician su actividad en el crepúsculo. El monitoreo de los mismos consistió en revisar las trampas cada dos horas a partir de su activación, haciendo una revisión a las 05:00 h.

Para la segunda metodología denominada Chacu se convocó a los pobladores de cada comunidad y estudiantes a participar en el Chacu de liebre, llegando a un aproximado de 50 personas, el cual consistió en rodear las zonas donde tenían sus madrigueras las liebres, cada persona portaba un saco de polipropileno, que permitiría captura a la liebre. Esta actividad empezó desde las 06:00 a 10:00 h. en vista de que las liebres durante las primeras horas del día descansan en madrigueras ubicadas en las pampas y cerros. Aplicamos el análisis estadístico de Kruskal Wallis, utilizando el programa INFOSTAT. Las variables analizadas fueron: Comunidades, número de individuos de liebre, porcentaje de daño y costo económico de daño de cultivos.

\section{Resultados y discusión.}

Estimación de la densidad poblacional (ind/ha) de la liebre europea.

Se evaluó, según la geo referenciación realizada, un área total de $22.13 \mathrm{~km}^{2}$, comprendida en las comunidades de Churo, Karina y Luquina Chico. Los resultados señalan que hay diferencia estadísticamente significativa entre el tamaño poblacional de liebre europea en las comunidades evaluadas a partir del método de transecto fijo. Se determina que en la comunidad de Luquina Chico, la densidad poblacional es de $2.4 \mathrm{ind} / \mathrm{ha}$; en la comunidad de Churo $1.2 \mathrm{ind} / \mathrm{ha}$ $y$ en la comunidad de Karina $1.11 \mathrm{ind} / \mathrm{ha}$ $\left(\mathrm{H}_{\text {calc }(0.05)}=12.01 ; 2 \mathrm{gl} ; \mathrm{p}=0.0039\right)$.

El resultado de densidad poblacional en la comunidad de Luquina Chico se debe principalmente a la disponibilidad de alimento que existe en este hábitat, siendo los cultivos de mayor producción cebada, avena, 
alfalfa, quinua y papa, de esta forma las liebres tienen alimento disponible casi durante todo el año por los 2 tiempos de siembra que se realiza en estas comunidades favorecido por el micro clima existente en los mismos. Al igual que Smith et al. (2005), manifiesta que la mayor cantidad de individuos de liebre se encuentra en los cultivos agrícolas. Actualmente, no hay cacería ni depredadores naturales para la liebre europea, está es la situación de incremento exponencial de la población de liebre.

El registro de huellas de liebre europea no presenta diferencia significativa entre las tres comunidades evaluadas, siendo mayor la cantidad de huellas registradas en la comunidad de Luquina Chico, mientras que en la comunidad de Karina se registró menor número de huellas

$\left(\mathrm{H}_{\text {calc }(0.05)}=1.55 ; 2 \mathrm{gl} ; \mathrm{p}=0.4606\right)$ evaluados $($ Tabla 1$)$ La prueba de contraste indica que las tres comunidades son similares en relación a la cantidad de huellas registradas. El registro de huellas de liebre europea presenta diferencia entre los cinco cultivos evaluados siendo mayor la cantidad de huellas en el cultivo de alfalfa encontrando 17 huellas, mientras que el menor número fue de 5 huellas, registrado en el cultivo de quinua $\left(\mathrm{H}_{\text {calc }(0.05)}=11.83 ; 4 \mathrm{gl} ; \mathrm{p}=0.0184\right)$.

Tabla 1. Promedio de huellas de liebre europea registradas en las estaciones olfativas actividades en los 5 cultivos evaluados en las comunidades de Churo, Karina y Luquina Chico de la península de Chucuito, lago Titicaca, Puno (diciembre 2010 a mayo 2011).

\begin{tabular}{lcccrc}
\hline Comunidad & Alfalfa & Cebada & Avena & Papa & Quinua \\
\hline Churo & 13.7 & 12.2 & 10.8 & 10.3 & 6.8 \\
Karina & 12.0 & 10.8 & 10.0 & 8.2 & 5.3 \\
Luquina & 16.8 & 13.8 & 12.0 & 9.2 & 6.5 \\
\hline
\end{tabular}

Este resultado se debe principalmente a la preferencia de la dieta alimenticia de la liebre europea basada en esta leguminosa (alfalfa) y la menos se registra en el cultivo de quinua con 5.3 individuos. Sin embargo, Canales (2008) menciona que el mayor porcentaje de daño que ocasiona la liebre europea es en el cultivo de alfalfa (30\%), cebada (28\%), mientras que el cultivo con menor daño es papa $(8 \%)$ y quinua $(0 \%)$.

El registro de huellas de liebre europea en la comunidad de Luquina Chico, si presenta diferencia entre los seis meses evaluados, siendo mayor la cantidad de huellas en diciembre, mientras que el menor número de huellas se obtuvo en abril $\left(\mathrm{H}_{\text {calc }(0.05)}=9.30 ; 4 \mathrm{gl} ; \mathrm{p}=0.0944\right)$. Los registros de huellas permiten evidenciar la abundancia o densidad poblacional, siendo un método indirecto de estimación poblacional, que se realizan, por ejemplo, en Chile con metodologías empleadas frecuentemente en los estudios poblacionales es el conteo de huellas que aparecen en sus áreas de alimentación, el cual dio como resultado 50 liebres/ha, efectiva en un $60 \%$ (Murray et al., 2002).
Al respecto, Koetsier et al. (1990) ratifica en su estudio realizado en Argentina que la mayor fluctuación de liebres europeas fueron registradas en cultivos de alfalfa, leguminosas preferidas por la especie en su alimentación. De la misma forma otros cultivos son afectados por esta especie como la avena y ají páprika. En un estudio similar, Santiago (2007) manifiesta que la liebre europea causa daños considerables en cultivos de hortalizas (zanahorias), cereales (centeno), leguminosas (alfalfa) y frutales (durazno, manzana).

Porcentaje de daño agrícola causado por la liebre europea.

Los resultados señalan que hay una clara pérdida económica registrada según la metodología aplicada en los cultivos a causa de la liebre europea, principalmente en los cultivos de alfalfa, el cual registra un $22.1 \%$ de daño en la comunidad de Luquina Chico, seguido de un $15.7 \%$ en la comunidad de Churo y $15.4 \%$ en la comunidad de Karina, mientras que el cultivo menos afectado fue el de quinua, con un $5.56 \%$ en Luquina Chico, $3.2 \%$ en Churo y $2.8 \%$ en Karina.

De la misma forma, los resultados reflejan que existe un $r=0.67$, que significa un $67 \%$ de asociación entre las dos variables evaluadas para el daño agrícola que son el porcentaje de pérdida y los costos acorde a precio de mercado, según el área promediada de cultivos ramoneados por liebre. En la comunidad de Karina se observa un grado de asociación de $\mathrm{r}=0.64$, que representa un 64\% y Luquina Chico un $49 \%$ de asociación entre las dos variables consideradas durante la evaluación (diciembre 2010 - mayo 2011).

Estos resultados infieren que a mayor daño por la liebre, mayor será el daño económico por consumo de cultivos que posee las familias en las comunidades. Esta especie es invasora y afecta negativamente a la biodiversidad y las actividades económicas productivas, como lo demuestra la lucha contra la especie en Chile y Argentina (Lleellish et al., 2007); debido a sus consumos de cereales, pastizales, rastrojo y alfalfas tiene valores negativos en la producción (Rodríguez et al., 1997; Purroy, 2011). Esta especie no posee preferencia de hábitat, pudiendo estar presente en una diversidad de estos, desplazándose entre ellos por épocas de seca y lluviosa (Carro et al., 2010); sin embargo, prefieren los pastizales y zonas de cereales, según la época de producción (Purroy, 2011). Este comportamiento hace que las comunidades de la península de Chucuito tengan la presencia de liebres durante todo el año, perjudicando sus cultivos agrícolas, ya que las liebres prefieren zonas agrícolas, donde su densidad se incrementa (Lazo et al., 1992).

Esta especie está fuertemente ligadas al medio agrícola y ganadero (Palacios \& Ramos 1979), por tanto, es importante determinar factores para su manejo en cuanto a la disminución poblacional, no solamente en la península de Chucuito, sino en las diferentes comunidades rurales de la región de Puno. 
Las densidades de las liebres, están relacionadas con las tasas reproductivas de las liebres, que realizan durante todo el año (Fernández et al., 2008), el comienzo de la actividad sexual no depende de la época del año sino del momento en que se alcanza la talla adecuada (Fernández et al., 2010) y pueden alcanzar una producción potencial de 9.8 liebres por año (Hansen, 1992; Alves et al., 2002), está situación está permitiendo el incremento exponencial de las liebres en el altiplano peruano

Métodos de captura adecuados de liebre europea.

Aplicamos 2 metodologías: trampas Lazo y el Chacu que consiste en que los pobladores se organizan para capturar a las liebres. Esta metodología del Chacu, se ha realizado una sola vez en cada comunidad a partir de las 06:00 a 10:00 h. Por ejemplo, en la comunidad de Churo se ha capturado con 6 individuos, en Luquina Chico 7 individuos y Karina con 5 individuos. Con respecto a las trampas Lazo de las 160 colocadas en total en las comunidades, se pudieron atrapar 5 individuos en Churo, 4 en Karina y 5 en Luquina Chico, se consideró como atrapado, cuando la liebre, activó la trampa Lazo, en el $100 \%$ de los casos las liebres mordieron las cuerdas de nylon de la trampa y se liberaron solos.

Actualmente, esta especie no posee depredador, sin embargo, existe un proceso de aprendizaje por parte del zorro para cazar liebres en las comunidades en estudio. Esta misma situación se presenta en Argentina, donde existe ausencia de zorros que pudieran ser depredadores efectivos de la liebre (Jaksic, 1998), siendo las liebres una importante dieta de varios depredadores (Zanón et al., 2012).

\section{Conclusiones.}

Con el uso del método de estaciones olfativas se estimó que de los $23 \mathrm{~km}$ geo referenciados como área de estudio, en la comunidad de Luquina Chico hay una mayor presencia de liebre europea con 11.7 individuos a comparación de las demás comunidades evaluadas.

- Aplicando el método de transectos con el estimador de Hayne se obtuvo $1.2 \mathrm{ind} / \mathrm{ha}$ en la comunidad de Churo, 1.1 ind/ha en Karina y 2.4 ind/ha en la comunidad de Luquina Chico.

El cultivo que tuvo mayor pérdida económica fue la alfalfa en Luquina chico con $22.1 \%$, seguido de la cebada y avena y en rangos menores la papa y quinua con $5.7 \%$, la misma secuencia de daño en el tipo de cultivo tuvieron las 3 comunidades evaluadas.

- Hay un grado de asociación entre las dos variables porcentaje de pérdida económica y costos económicos, siendo $\mathrm{r}=0.67$ en Churo, $\mathrm{r}=0.64$ en Karina y r $=0.68$ en Luquina Chico.

\section{Agradecimientos.}

A las comunidades de Luquina Chico, Karina y Churo de la Península de Chucuito - Puno, por facilitar el acceso a sus cultivos.

\section{Literatura citada.}

Alves P. C., Goncalves H. Santos M. \& Rocha A. 2002. Reproductive biology of the Iberian hare, Lepus granatensis, in Portugal. Mammalian Biology, 67 (6): 358-371.

Bonino N., Cossios D. \& Meneghetti J. 2010. Dispersal of the European hare (Lepus europaaus) in South America. Folia Zoologica, 59: 9-15.

Bonino N.A., Pelliza M., Manacorda \& Larosa F. 1997. Food partitioning between the mara the introduced hare (Lepus europaeus) in the southern Monte desert, Argentina. Studies on Neotropical Fauna and Environment 32: 129134.

Canales G.A. 2008. Evaluación poblacional de Lepus europaeus Pallas, 1778 (liebre europea), sus efectos en la agricultura de la península de Capachica. Revista de Investigación (Esc. Post Grado UNA- Puno). V4. № 2 : 187-198.

Carro F., Beltrán J.F., Fernández A., Camacho I.M. \& Soriguer R.C. 2010. Bioquímica, hematología de las liebres en Doñana y condición física en Navarra y Doñana. 206-228.

Cossios D. 2004. La liebre europea, (Lepus europaeus) (Mammalia, Leporidae), especie invasora en el sur del Perú. Rev Perú. Biol. 11(2): 209-212.

Fernández A., Soriguer R., Carro F. \& Castién E. 2010. Relation between physical condition and reproductive activity in a population of Iberian hares, Lepus granatensis in northern Iberian Península. Folia Zoológica, 59 (1): 1-8.

Fernández A., Soriguer R., Castién E. \& Carro F. 2008. Reproduction parameters of the Iberian hare Lepus granatensis at the edge of its range. Wildlife Biology, 14 (4): 434-443.

Hansen K. 1992. Reproducción in European hare in a Danish Farmland. Acta Theriologica, 37: 27-40.

INTA. 2012. Instituto Nacional de Tecnología Agropecuaria"; Estación Experimental Agropecuaria Bariloche; Área Forestal, Proyecto Regional Argentina.

Jaksic F. 1998. Vertebrate invaders and their ecological impacts in Chile. Biodiversity and Conservation 7: 14271445.

Koetsier P.P., Dey G., Mladenka \& Check J. 1990. Rejecting equilibrium theory - a cautionary note. Bulletin of the Ecological Society of America 71: 229-231.

Lazo A., de le Court C. \& Soriguer R.C. 1992. Evaluation of hare abundance allowed by their use of attraction points. Zeitschrift fur Saugetierkunde, 57 (6): 373-379.

Lleellish M., Cadenillas R. \& Chipana G. 2007. Presencia de liebre europea (Lepus europaeus) en el Departamento de Tacna. Serie de Publicaciones de Flora y Fauna Silvestre. Instituto Nacional de Recursos Naturales, Lima, Perú.

Murray D.L., Roth J.D., Ellsworth E., Wirsing A.J. \& Steury T.D. 2002. Estimating low-density snowshoe hare populations using fecal pellet counts. Canadian Journal of Zoology, 80: 771-781.

Palacios F. \& Ramos B. 1979. Situación actual de las liebres en España y medidas para su conservación. Boletín de la Estación Central de Ecología, 8 (15): 69-75. 
Purroy F.J. 2011. Liebre ibérica - Lepus granatensis. En: Enciclopedia Virtual de los Vertebrados Españoles.

Rodríguez M., Palacios J., Martín J.A., Yanes T., Martín P., Sánchez C., Navesco M.A., Muñoz R. \& Yanes T. 1997. La Liebre. Ediciones Mundi-Prensa, Madrid.

Salomone F. 2006. La liebre europea en Valles y Altiplano de Bolivia. Ediciones CIPCA, La Paz, Bolivia.

Santiago G. 2007. Especies exóticas: algunos ejemplos en la Argentina. La Plata, Argentina.

Smith R.K., Jennings N.V., Robinson A. \& Harris S. 2005. Conservation of European hares (Lepus europaeus) in Britain: is increasing habitat heterogeneity in farmland the answer? J. Appl. Ecol.41(6): 1092-1102.

Vu A. 2001. Lepus europaeus, Animal Diversity. Web [en línea].

(http://animaldiversity.ummz.umich.edu/site/accounts/in formation/Lepus europaeus.html) Consulta: 23 de agosto del 2003.
Wiens J.A. 1984. On understanding a non-equilibrium world: myth and reality in community patterns and processes. 439-457, en D.R. Strong, D. Simberloff, L.G. Abele y A.B. Thistle, eds. Ecological Communities. Conceptual Issues and the Evidence. Princeton University Press, Princeton.

Zanón M.J.I., Travaini A., Zapata S., Procopio D. \& Santillán M.A. 2012. The ecological role of native and introduced species in the diet of the puma (Puma concolor) in southern Patagonia. Oryx, 46(1): 106-111.

Zeballos H., Medina C., Pino K., Mejía- Ríos A. \& Pari A. 2012. La liebre europea (Lepus europaeus) (Lagomorpha: Leporidae) una especie invasora en el Perú. Rev Perú. Biol, 19: 267-273.

\footnotetext{
${ }^{1}$ Biólogo CEDESOS Puno.

${ }^{2}$ Biólogo, Docente Facultad de Ciencias Biológicas de la Universidad Nacional del Altiplano Puno. acanales7@hotmail.com.
} 